\title{
Weight loss at your fingertips - personalized nutrition using fasting glucose and insulin
}

\author{
Christian Ritz, Arne Astrup, Thomas M. Larsen and Mads F. Hjorth \\ Department of Nutrition, Exercise and Sports, Faculty of Sciences, University of Copenhagen, Copenhagen, Denmark
}

\begin{abstract}
Introduction: Precision medicine is changing the way people are diagnosed and treated into a more personalized approach. In medical research, several statistical methods have been proposed for estimating personalized treatment effects. However, in nutritional science these methods have hardly been used. By re-evaluation of pre-treatment biomarker data, we demonstrate how two diets cause differential weight loss depending on pre-treatment fasting plasma glucose (FPG) and fasting insulin (FI) levels.
\end{abstract}

Materials and Methods: Overweight people with increased waist circumference were randomly assigned to receive an ad libitum New Nordic Diet (NND) high in dietary fiber and whole grain or an Average Danish (Western) Diet (ADD) for 26 weeks. All foods were provided free of charge. Body weight was measured throughout the study and blood was drawn before randomization from where FPG and FI were analyzed. Weight was described by linear mixed models including biomarker-diet group interactions, covariate adjustment, and participant-specific random effects. Personalized predictions of additional weight loss from NND compared to ADD given specific values of FPG or FI were estimated as contrasts of intercepts and slopes obtained from the biomarker-diet group interaction term.

Results: Baseline FPG predicted a $3.00(1.18 ; 4.83, \mathrm{n}=181, \mathrm{P}=0.001) \mathrm{kg}$ larger weight loss per mmol/L from choosing NND over ADD. For instance, a baseline FPG level of $4.7 \mathrm{mmol} / \mathrm{L}$ would lead to an average of $1.42 \mathrm{~kg}$ larger weight loss on NND vs. ADD (above $0.41 \mathrm{~kg}$ with $95 \%$ certainty), whereas the average effect size would be $8.33 \mathrm{~kg}$ (above $5.50 \mathrm{~kg}$ with $95 \%$ certainty) among subjects with FPG level of $7.0 \mathrm{mmol} / \mathrm{L}$. Among individuals with FPG $<5.6 \mathrm{mmol} / \mathrm{L}$, each pmol/L lower baseline FI predicted a $0.039(95 \%$ CI $0.017 ; 0.061, \mathrm{n}=143, \mathrm{P}<0.001) \mathrm{kg}$ larger weight loss from choosing NND over ADD. For instance, a baseline FI level of $25 \mathrm{pmol} / \mathrm{L}$ would lead to an average larger weight loss of $4.10 \mathrm{~kg}$ on NND vs. ADD (>2.51 kg with $99 \%$ certainty). Likewise, a baseline FI level of $75 \mathrm{pmol} / \mathrm{L}$ would result in an average effect size of $2.15 \mathrm{~kg}(>1.11 \mathrm{~kg}$ with $99 \%$ certainty).

Discussion: Use of pre-treatment FPG and FI led to truly individualized predictions of treatment effect of introducing more fiber and whole grain in the diet on weight loss, ranging from almost no effect to losing more than $8 \mathrm{~kg}$. These findings tentatively suggest that re-evaluation of data from existing randomized controlled trials through suitable statistical methods may have a great potential.

\section{Conflict of Interest}

$\mathrm{CR}, \mathrm{AA}$, and MFH are co-inventors on a pending provisional patent application for the use of biomarkers to predict responses to weight loss diets owned by UCPH, in accordance with Danish law. AA is consultant/ member of a number of advisory boards. MFH and AA are co-authors of books about personalized nutrition for weight loss published by Politikens Forlag, Denmark. MFH and AA are co-founders and co-owners of the UCPH spin-off Personalized Weight Management Research Consortium ApS (http://Gluco-diet.dk). TML serves as advisor for Sense diet program. 